\title{
Microstructure Characterization of an Ultra-Fast Heated Medium Carbon Chromium-Manganese High Strength Steel
}

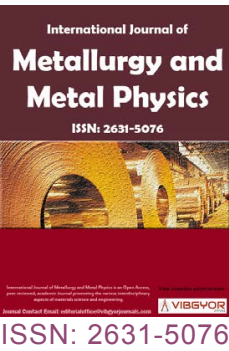

\section{A Banis* and S Papaefthymiou}

\section{Laboratory of Physical Metallurgy, National Technical University of Athens, Greece}

\begin{abstract}
The purpose of this work is to create mixed phased advanced high strength microstructures based on an ultra-fast heat treatment. The current work focuses on a hot rolled $42 \mathrm{CrMn} 6$ steel with medium-carbon and chromium - manganese additions. In order to better understand the microstructure evolution and the phenomena that take place during rapid heating, an ultra-fast heated sample was analyzed and compared with a conventionally treated sample. The methods employed for the microstructure characterization included Scanning Electron Microscopy (SEM) with Electron Back-Scatter Diffraction (EBSD), Transmission Electron Microscopy (TEM) and hardness testing. The initial microstructure of both samples consisted of ferrite and spheroidized cementite. The conventional heat treatment resulted in a fully martensitic microstructure as expected. On the other hand, the ultra-fast heated sample showed greater heterogeneity in its final microstructure. This was the result of the lack of time necessary for carbon diffusion, cementite dissolution and chemical composition homogenization at the austenitic range. Its final microstructure consisted of undissolved spheroidized cementite, nano-carbides and martensite laths on a ferritic matrix. Based on the TEM analysis, traces of bainitic ferrite are indicated. The grains and lath size observed in SEM - TEM and as derived from the EBSD analysis, offer proof that a diffusionless, massive transformation takes place for the austenite formation and growth instead of a diffusion-controlled transformation that occurs on a conventional heat treatment.
\end{abstract}

\section{Keywords}

Ultra-fast heating, Bainite, Diffusionless transformations, AHSS

\section{Introduction}

In order to improve the major steel properties, such as strength and ductility, two basic principles are used: (a) Alloying steel with proper elements (e.g. nickel - $\mathrm{Ni}$, manganese - $\mathrm{Mn}$, chromium - $\mathrm{Cr}$, molybdenum - Mo etc.), which dramatically increase the material cost and adversely affect the viability of the raw materials; and (b) Excessive re- fining of the grains, as applied in thin sheets/plates obtained with fully controlled thermo-mechanical rolling (e.g. Thermo-mechanical controlled processing - TMCP), which offers a uniform distribution of grain boundaries. Thus, the combination of the grain refinement effect with a specific and desirable distribution of micro-constituents (ferrite, bainitic ferrite, martensite, residual austenite and

*Corresponding author: A Banis, Laboratory of Physical Metallurgy, Division of Metallurgy and Materials, School of Mining \& Metallurgical Engineering, National Technical University of Athens, 9, Her. Polytechniou str., Zografou, 15780 Athens, Greece

Accepted: October 23, 2018; Published: October 25, 2018

Copyright: (C) 2018 Banis A, et al. This is an open-access article distributed under the terms of the Creative Commons Attribution License, which permits unrestricted use, distribution, and reproduction in any medium, provided the original author and source are credited.

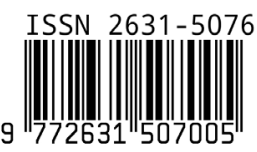

Banis and Papaefthymiou. Int J Metall Met Phys 2018, 3:021 
carbides) in the final microstructure is considered to positively affect both the strength and ductility of advanced high strength steels without the need for alloying with high concentrations of costly alloy additives (e.g. $\mathrm{Ni}, \mathrm{Mn}, \mathrm{Mo}, \mathrm{Cr}$ ).

Therefore, the need for new, cost-efficient methods to produce AHSS with good combination of strength and ductility is very important and hence, the ultra-fast heat treatment was introduced by Cola, et al. [1,2]. This treatment has been widely used before in the case-hardening of medium carbon steels [3]. However, its application to low carbon sheet steels is relatively new. A yield stress of 1.2-1.5 GPa has been reported for a $0.2 \% \mathrm{C}$ steel subjected to the 'Flash Bainite process'. These values are much higher than the yield stress obtained for the same material subjected to conventional heating rates. Furthermore, a $200 \mathrm{MPa}$ improvement in the yield stress has been reported for quenched and partitioned (Q\&P) and dual-phase (DP) steel grades subjected to ultra-fast heat treatment. This treatment affects the microstructure during recrystallization and phase transformation and affects their interaction to obtain better mechanical properties [4]. Several conditions need to be met in order to have a strong refinement and hence better properties. It has been shown that very high heating rates above $2000^{\circ} \mathrm{C} / \mathrm{s}$ are practically inapplicable while initial studies show that the effect of significant grain refinement and increase in strength and elongation can already be achieved at more realistic rates between 200-1000 ${ }^{\circ} \mathrm{C} / \mathrm{s}[1,3,5-8]$. In addition, the complex interactions between recrystallization, phase transformations and dissolution of carbides are not well described or controlled. The balance between these interactions will be the key to achieving an excellent combination of properties.

The influence of heating rates on the microstructure can be discussed in two main contexts. On the one hand is the effect of the heating rate on the recrystallization of a cold and hot rolled steel; while on the other hand is the effect of heating rate on phase transformations $[1,3,6,9,10]$. It is well documented [11] that the interaction between the two processes occurs when heating rates exceed certain limits, which depend to a large extent on the chemical composition of the steel and the degree of hot or cold deformation. Some of the basic theoretical aspects of the ultra-fast heating of carbon steel were summarized by Meshkov and
Pereloma [12]. Specifically, they elaborated on the effect of the initial microstructure on the austenite formation mechanism and concluded that the nucleation step can be achieved either by diffusion or diffusionless mechanisms and the growth stage can be controlled by either diffusion or interfaces (massive transformation). This is in accordance to Cerda, et al. [13]. As for the diffusionless mechanisms, Kaluba, et al. [14-16] supported a so-called bainitic transformation mechanism to form austenite when very high heating rates are applied. It is known that the initial microstructure, determined by the composition of steel and its hot/cold deformation during rolling, significantly affects recrystallization and overall behavior during phase transformations. This applies even more for the conditions of ultra-fast heating.

The effect of heating rate on phase composition and volume fraction of individual microconstituents was also studied [17]. Ultra-fast heating of a low-carbon steel to intercritical peak temperature leads to a complex microstructure of the ferritic matrix, which consists of recrystallized and recovered grains [10]. Therefore, the local mechanical behavior of the ferritic matrix might dramatically vary depending on the local dislocation density.

According to Puype [18], ultra-fast heat treatment offers the ability of microstructure refinement by suppressing the recrystallization. The reason is that this treatment influences the bifurcation of the recovery processes and reduces the driving force for recrystallization together with the limitation of growth by carbides and the interaction of solute elements with dislocations. The recrystallization hence starts at higher temperatures where the number of nuclei will be higher resulting in fine recrystallized grains. As phase transformation starts before the end of recrystallization, more nucleation sites for austenitization are available leading to a finer non-equilibrium transformed product. The growth of these fine recrystallized grains will be limited as dislocations are pinned by solutes such as precipitated carbides in the grain boundaries. Therefore, the phase transformation is enabled to occur in a partial recrystallized matrix, providing more nucleation sites for the transformation to austenite. Due to the increased number of nucleation sites for phase transformation, the grain size will be decreased after the double $\alpha \rightarrow \gamma \rightarrow \alpha$ transformation. 
Recently, Papaefthymiou, et al. $[19,20]$ showed the effect of the heating rate on the evolution of the microstructure of a slightly annealed medium carbon steel which rationalized the kinetics of the dissolution of cementite and the formation of austenite. The experimental results were compared with calculations in Dictra and the potential effects on the austenite-controlled diffusion kinetics were also evaluated. The application of a conventional heat treatment $\left(20^{\circ} \mathrm{C} / \mathrm{s}\right)$ and an ultra-fast $(300$ ${ }^{\circ} \mathrm{C} / \mathrm{s}$ ) to a steel with an initial microstructure consisting of ferrite and spheroidized carbides showed a remarkable difference in the obtained microstructures. After the conventional treatment the microstructure consists of martensite with fine carbides [21]. Following the ultra-fast treatment, the microstructure consists of nanostructured martensitic laths, large spherical carbides and $\mathrm{M}_{7} \mathrm{C}_{3}$. The fine carbides are located between BCC laths, which may indicate presence of bainite. In addition, Papaefthymiou and Goulas $[19,22]$ concluded that the mixed microstructure product of ultra-fast heat treatment constitutes indirect evidence of the presence of carbon gradients in austenite and a contrast rim is produced, which is attributed to the heterogeneous distribution of alloying elements in austenite during cementite dissolution.

The purpose of the present work is therefore the characterization of the microstructure evolution during an ultra-fast heat treatment on a medium carbon steel. In order to understand the transformation and diffusion phenomena that take place during the heating process, a conventional heat treatment was used as reference. According to the state of art, the lack of heating time leads to a diffusionless transformation mechanism and prevents the dissolution of carbides and other solutes. These are expected to act as nucleation sites resulting in a very fine microstructure, with increased dislocation density. Also, the undissolved carbides and lack of heating time might impede the recovery and recrystallization of some very fine ferrite grains thus preventing their transformation to austenite and being present in the final microstructure. In order to confirm these, SEM and TEM were used to observe the micro-constituents and STEM/EDS for their chemical analysis while EBSD was used to study the dislocation density and the grain sizes. An initial effort is also made to correlate the microstructure with the mechanical properties of the steel and especially its hardness.

Table 1: The chemical composition of the researched steel. It is a medium carbon $42 \mathrm{CrMn} 6$ hot rolled steel.

\begin{tabular}{|l|l|l|l|l|l|l|l|l|}
\hline C (\%wt.) & Si (\%wt.) & Mn (\%wt.) & S (\%wt.) & P (\%wt.) & Cr (\%wt.) & Mo (\%wt.) & Ti (\%wt.) & N (\%wt.) \\
\hline 0.43 & 0.4 & 1.43 & 0.0135 & 0.013 & 1.23 & 0.1 & $<0.05$ & $0.01-0.02$ \\
\hline
\end{tabular}

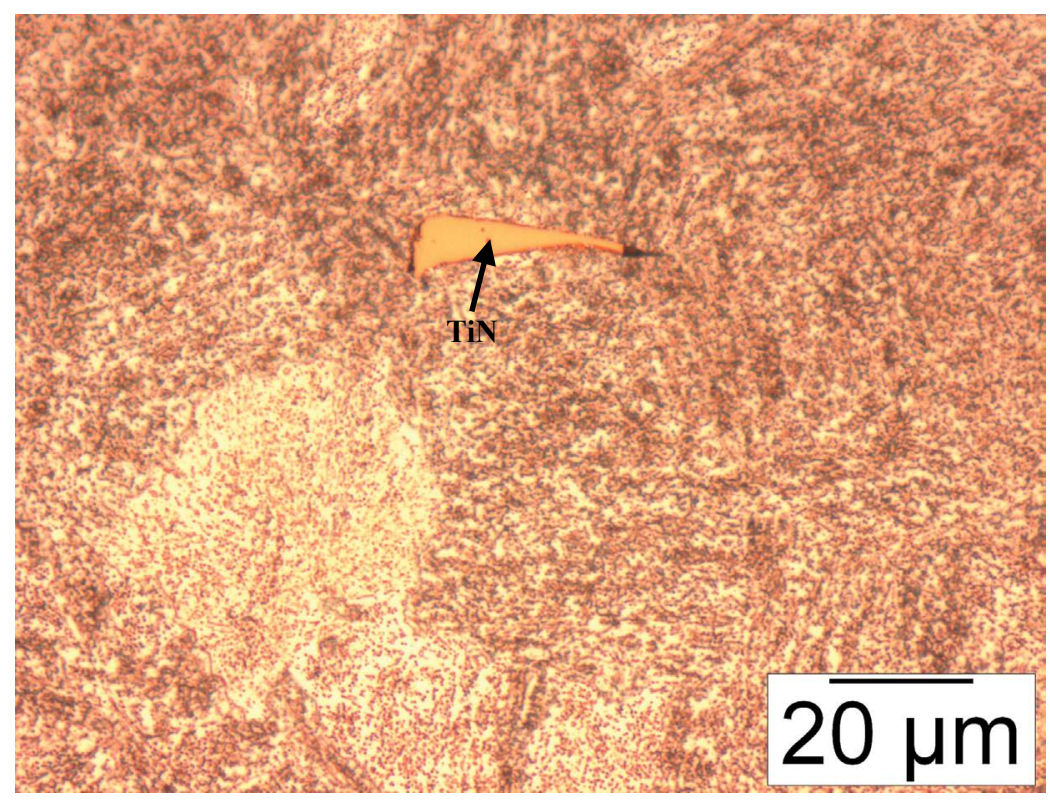

Figure 1: LOM image of the initial microstructure of the steel used before heat treatment. The microstructure was obtained by thermomechanical treatment and consists of ferrite and spheroidized cementite. A TiN is also observed in the initial microstructure. 


\section{Materials and Experimental Procedure}

In order to examine the microstructure evolution under ultra-fast heat treatment a hot-rolled, medium carbon, chromium manganese enriched steel $(42 \mathrm{CrMn} 6)$ was selected. The chemical composition can be seen in Table 1. The initial microstructure of both samples consisted of ferrite and spheroidized cementite as seen in Figure 1. Controlled-heating experiments were performed in a Bähr 805A Quench dilatometer. Dilatometric plate and round samples (plate sizes length $10 \mathrm{~mm}$ with width $2 \mathrm{~mm}$; round sizes: Length $10 \mathrm{~mm}$ with diameter of $3 \mathrm{~mm}$ ) derived from cross sections cut out of hot rolled bars with dimensions $95 \times 49 \times$ $5500 \mathrm{~mm}^{3}$ (width $\times$ thickness $\times$ length) were used for the dilatometric experiments. The temperature was controlled by an S-type thermocouple spot welded to the midsection of each test sample [23]. The thermal cycle that was used for the ultra-fast heat-treated sample (UFHS) and the conventional heat-treated sample (CHS) is shown in Figure 2.

A very fast heating rate of $300^{\circ} \mathrm{C} / \mathrm{s}$ was selected for the UFHS, in order to prevent carbide dissolution and diffusion while the short soaking time of 2 $s$ does not allow the growth of the austenite grains. The peak Temperature $\left(T_{p}\right)$ of $1080^{\circ} \mathrm{C}$ is higher than the $A_{3}$ and therefore a fully austenitic microstruc- ture is expected in this temperature. Helium was used for quenching to ensure very fast cooling rates until room temperature (bellow $M_{s}$ ) is reached. For comparison reasons, another sample, with the same initial microstructure, was examined and was subjected to a conventional heat treatment. This treatment included a heating rate of $10^{\circ} \mathrm{C} / \mathrm{s}$, which ensures enough time for ferrite recrystallization and for Carbon diffusion. The $\mathrm{T}_{\mathrm{p}}$ was $900{ }^{\circ} \mathrm{C}$, which is slightly higher than the $A_{3}$, so a fully austenitic microstructure is also expected. The soaking time of $360 \mathrm{~s}$ (6 min) is enough for the austenite grains to grow. Helium quenching was also used to ensure the martensitic transformation.

Samples taken from each tested specimen were prepared, in accordance with standard procedure, by grinding at $280,400,800,1200,2000$ grid papers and polishing to a mirror-like finish using $6 \mu \mathrm{m}$ and $1 \mu \mathrm{m}$ diamond pastes and etching in a solution of $2 \% \mathrm{v} / \mathrm{v} \mathrm{HNO}_{3}$ in ethanol (Nital 2\%) for $5 \mathrm{~s}$ to reveal the microstructure. For TEM analysis disk specimens were cut from the dilatometry samples, manually ground to a thickness of $20 \mu \mathrm{m}$ and then the final thinning was carried out with Precision Ion Polishing System (PIPS).

For SEM analysis a JEOL6380LV SEM with mounted EDS and EBSD cameras was used while a Jeol

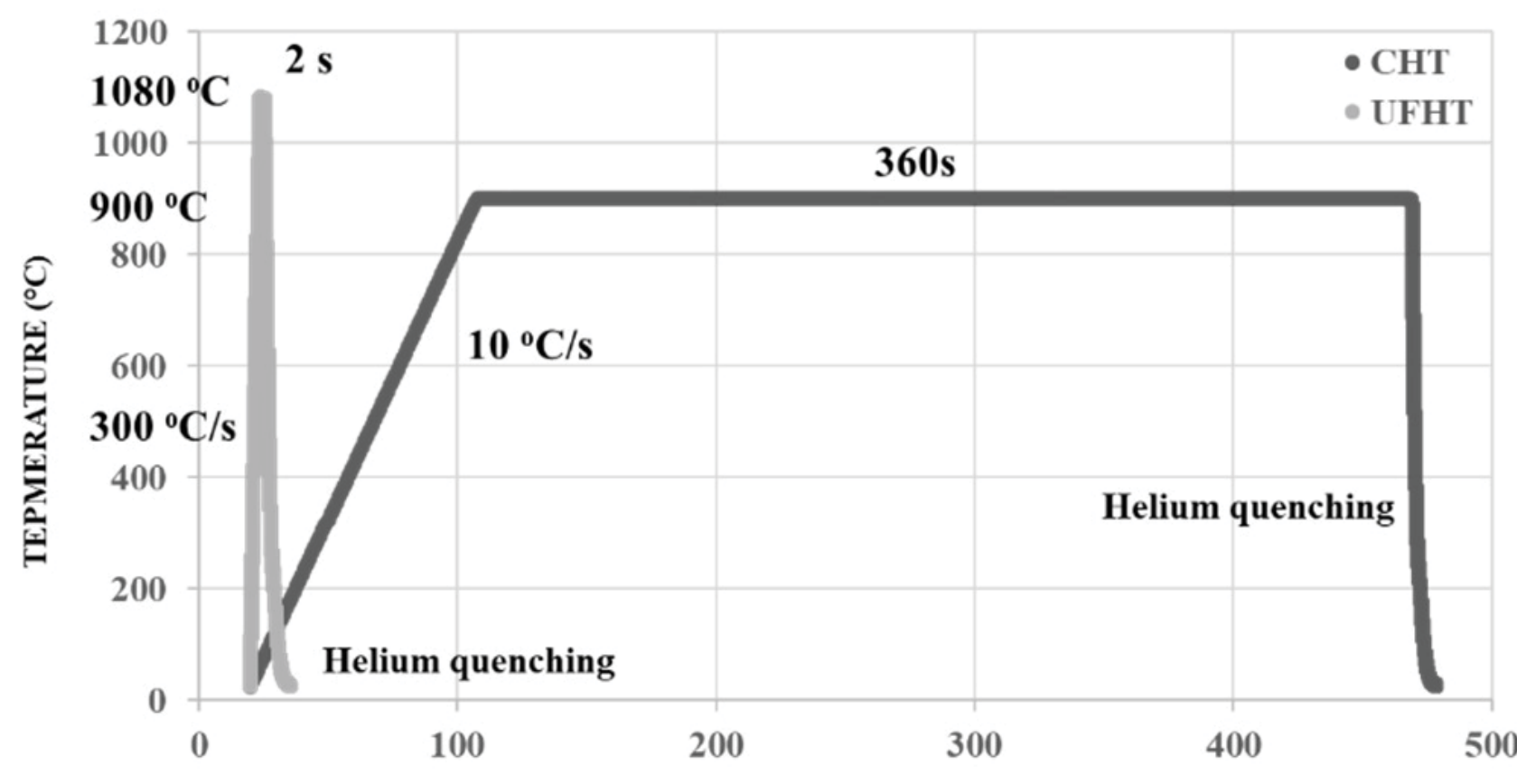

TIME (sec)

Figure 2: Time-Temperature diagram of the two heat treatments that were conducted. The ultra-fast heat treatment has a much smaller duration than the conventional heat treatment. 


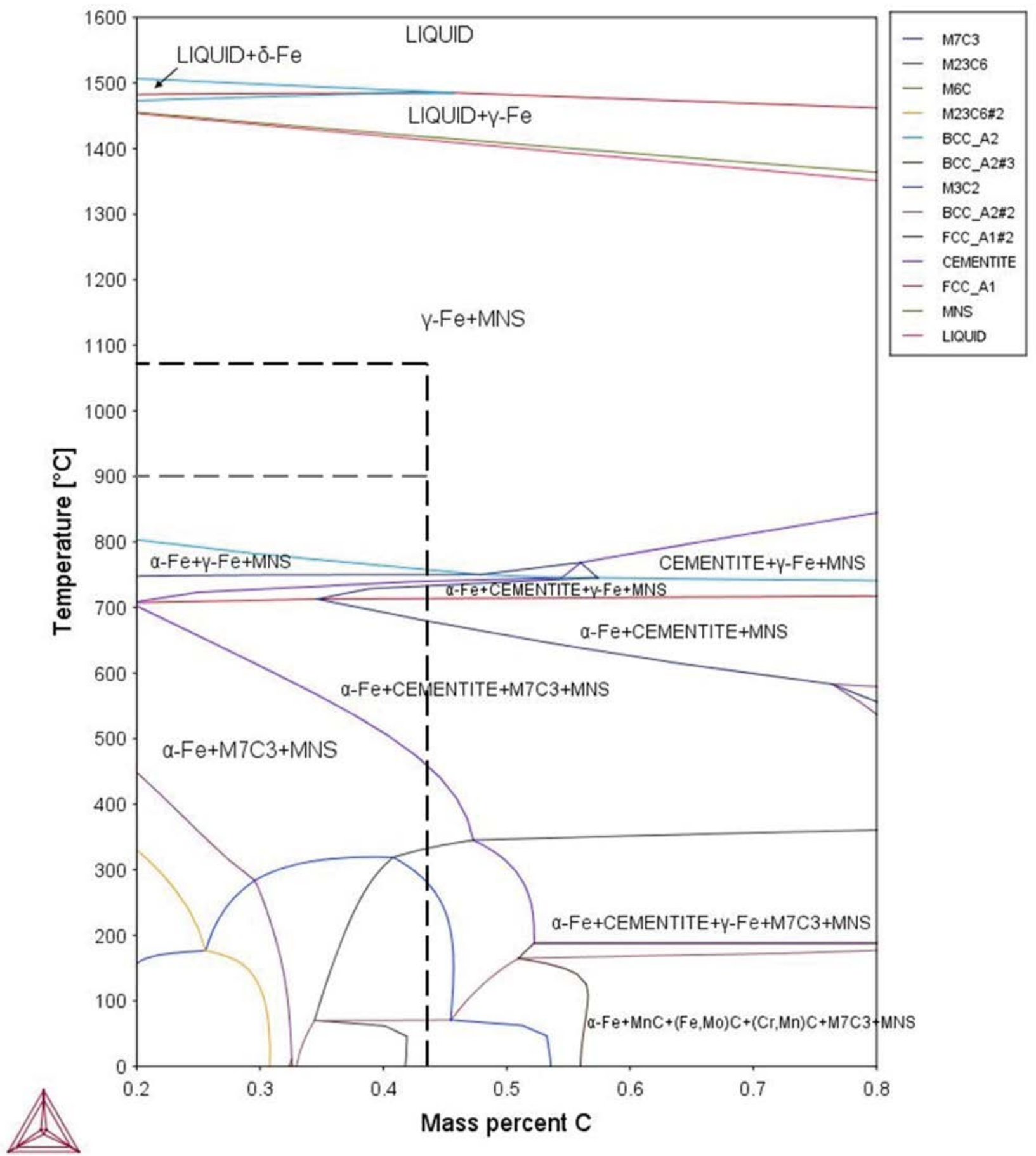

Figure 3: The phase transformation diagram of the materials used, as calculated from Thermocalc. The carbon content of both samples and the $\mathrm{T}_{\mathrm{p}}$ temperatures for the CHS and UFHS are also shown.

$2100 \mathrm{HR}, 200 \mathrm{kV}$ TEM mounted with EDS and STEM detectors was used for the TEM analysis.

For the hardness tests, Vickers Hardness (HV) test was selected with a diamond indent $\left(136^{\circ}\right.$ angle) and a load of $196 \mathrm{~N}$.

\section{Results and Discussion}

\section{Thermocalc results}

Thermocalc simulation software was used to produce the phase diagram of the material according to the chemical composition (Figure 3). According to the phase diagram, the $\mathrm{A}_{1}$ temperature is above $700{ }^{\circ} \mathrm{C}$ while the $A_{3}$ is below $800{ }^{\circ} \mathrm{C}$. The UFHS had a $\mathrm{T}_{p}$ of $1080^{\circ} \mathrm{C}$ which is much higher than the $\mathrm{A}_{3}$ and from the diagram can be expected that all of ferrite has transformed to austenite and MnS should exist in the microstructure. The transformation temperatures generated from the diagram are used as reference because the simulation software 


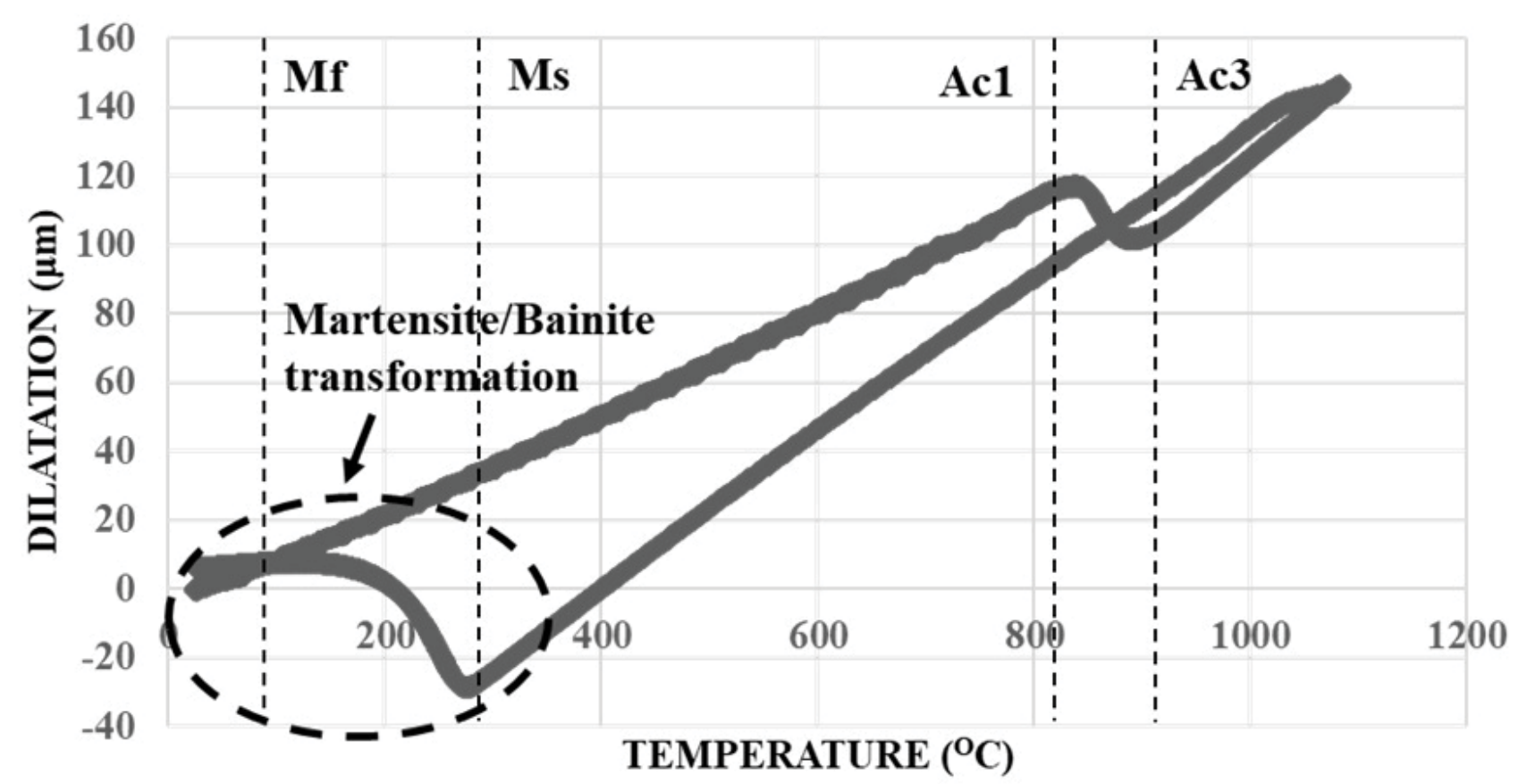

Figure 4: Temperature-Dilatation diagram derived from the dilatometry treatment. The transformation temperatures $\left(\mathrm{Ac}_{1}, \mathrm{Ac}_{3}, \mathrm{M}_{\mathrm{s}}, \mathrm{M}_{\mathrm{f}}\right)$ can be calculated from this diagram.

calculates the temperatures in equilibrium while the transformations that take place in the selected heat treatments are in para-equilibrium because of the ultra-fast heating rates.

\section{Dilatometry results}

Dilatometry results show the temperatures of the transformations that take place during the ultra-fast heating. According to Valdes-Tabernero, et al. $[24,25]$, with increasing heating rates to the ultrafast range, there is a shift in the $\mathrm{Ac}_{1}$ and $\mathrm{Ac}_{3}$ temperatures to higher values, so it is expected that the temperatures of $810^{\circ} \mathrm{C}$ and $900{ }^{\circ} \mathrm{C}$ that are calculated from the dilatometry are higher than those expected from a conventional heat treatment. Specifically, as shown in Figure 4 the austenite transformation indeed starts at $810{ }^{\circ} \mathrm{C}\left(\mathrm{Ac}_{1}\right)$ and ends at $900{ }^{\circ} \mathrm{C}\left(\mathrm{Ac}_{3}\right)$ which are higher than these calculated from the simulation phase diagram. Also, the martensite transformation during quenching starts at $290{ }^{\circ} \mathrm{C}\left(\mathrm{M}_{\mathrm{s}}\right)$ and ends at $110{ }^{\circ} \mathrm{C}\left(\mathrm{M}_{\mathrm{f}}\right)$. The change in dilatation during the transformation can be explained crystallographically. During the austenite transformation, ferrite which has BCC lattice transforms to austenite with FCC lattice. The atomic packing factor (APF) of these lattices is 0.68 and 0.74 respectively. Therefore, this can explain the decrease in the dilatation during austenite transformation. On the other hand, during marten- site transformation, austenite (FCC) transforms to martensite or bainite (BCT atomic packing factor: 0.687) [26], thus there is a reduction in the APF and therefore an increase in the dilatation. Also, the presence of substitutional alloying elements $(\mathrm{Cr}, \mathrm{Mn}, \mathrm{Mo})$, as seen in Table 1 , leads to the retardation of the bainitic transformation to temperatures lower than the Ms [27]. Therefore, the bainitic transformation cannot be observed in the dilatation diagrams, as it takes place alongside the martensitic transformation. Finally, Castro Cerda, et al. reported that above the $\mathrm{Ac}_{3}$ temperature, the carbon diffusion-controlled austenite formation during conventional heat treatment is replaced by a massive mechanism of austenite growth upon ultra-fast heat treatment [28].

\section{SEM results}

The microstructure of the conventional heat-treated steel is shown in Figure $5 a$, Figure $5 c$ and Figure $5 e$. It is a fully martensitic microstructure with no evidence of cementite or undissolved carbides. The martensite lath size shows no deviations and it is larger than the average lath size of the ultra-fast heated steel. On the other hand, the SEM results of the ultra-fast heated sample show complex microstructures with great heterogeneity concerning the grain size and the microstructure components. It can be clearly seen that the spheroidized cemen- 
tite that existed in the initial microstructure has not been dissolved because of the lack of time for diffusion. Some Prior Austenite Grain Boundaries (PAGBs) can be seen that indicate that the austenite grains before quenching had very dissimilar siz- es (Figure 5b). The difference in the local chemical composition [25] because of the short heating time and the difference of austenite grain size prior to quenching are important factors that lead to different results after quenching. As seen in Figure $5 d$
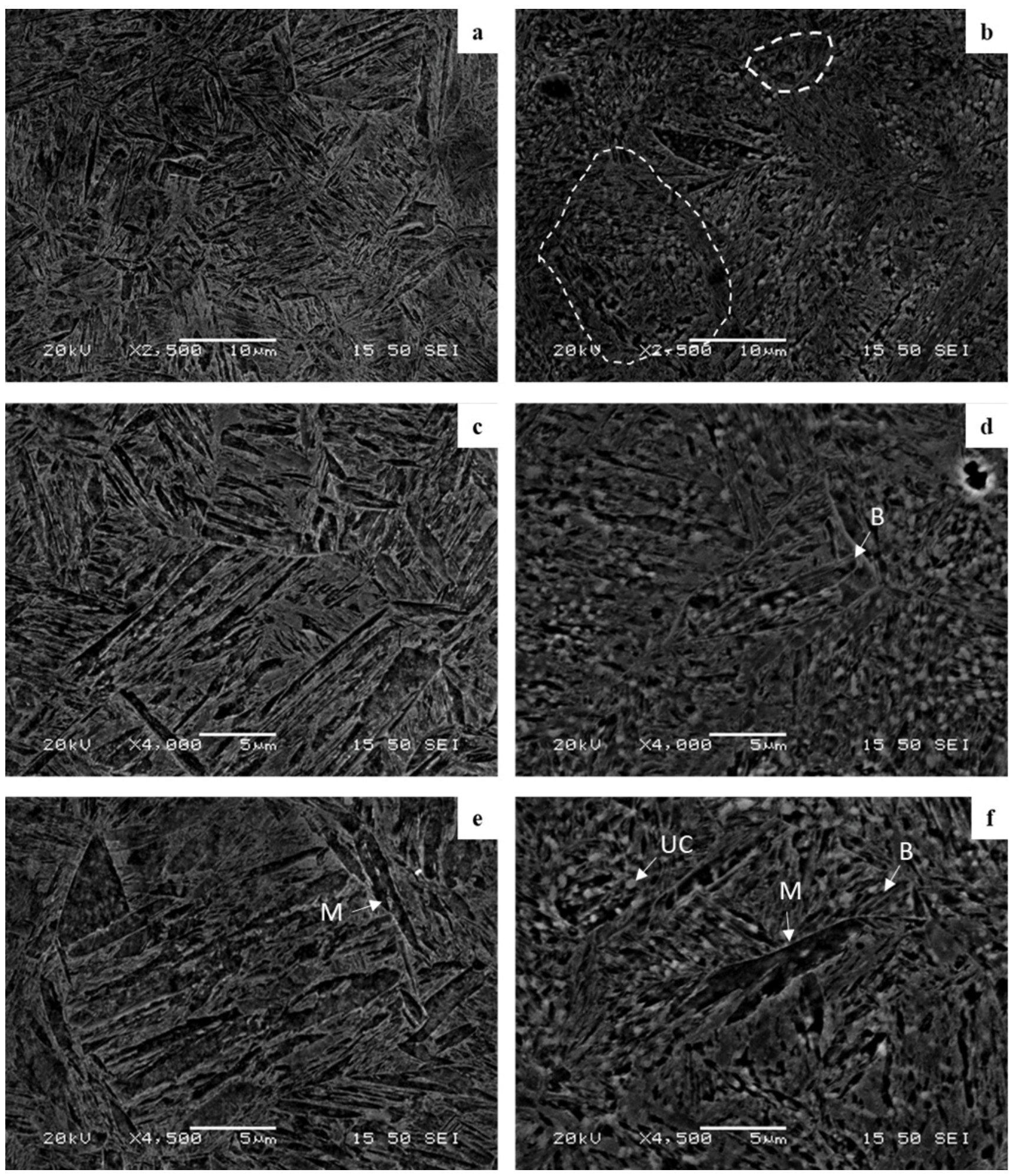

Figure 5: SEM images of the CHS $(a, c, e)$ and the UFHS $(b, d, f)$. The CHS consists of a fully martensitic microstructure while the UFHS microstructure indicates the existence of martensite (M), undissolved cementite (UC) and bainite (B). 


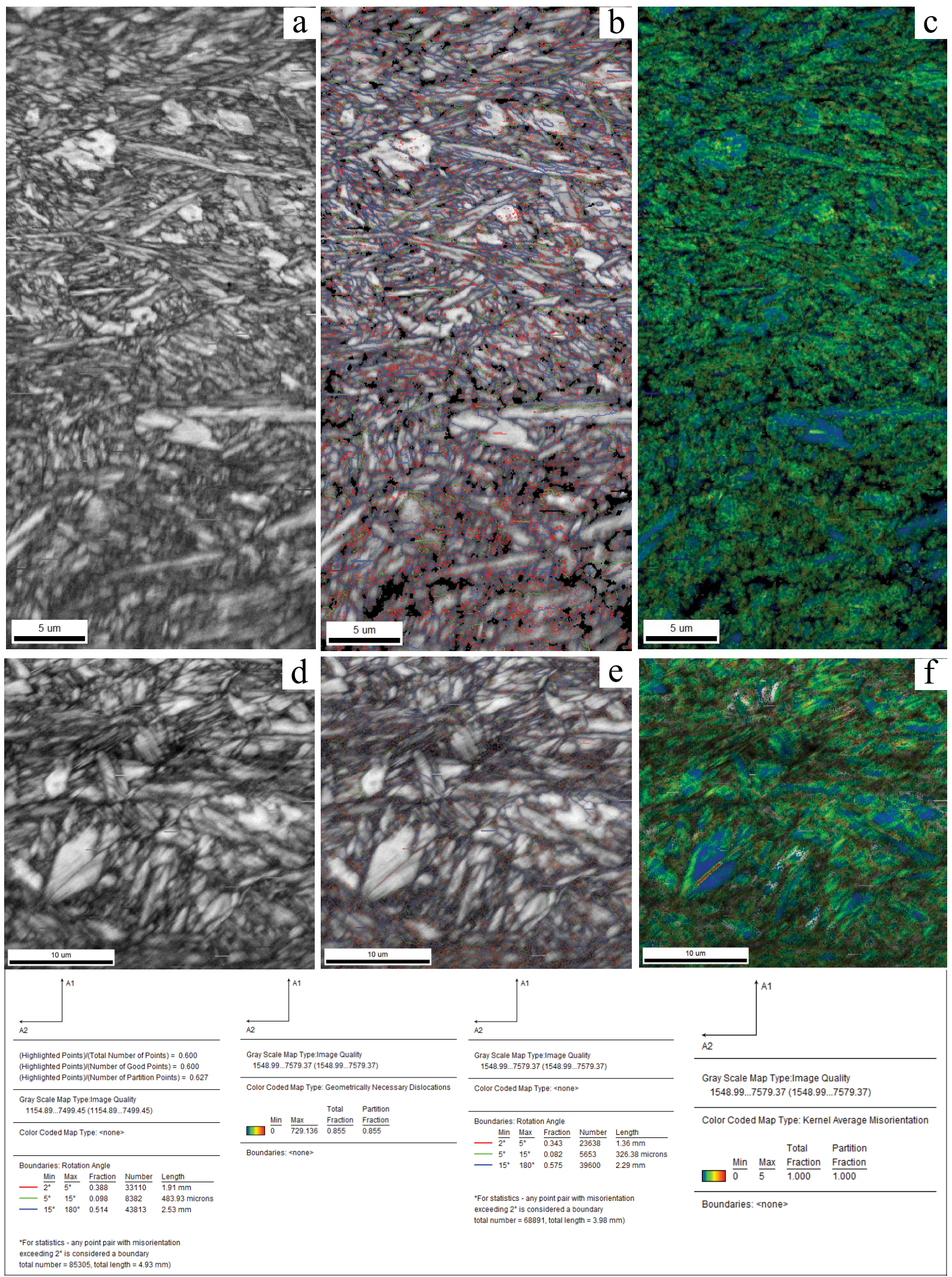

Figure 6: a) IQ map with the microstructure of the UFHS. Different martensite lath sizes can be easily observed; b) Misorientation map of the same area of the sample. Blue color indicates HAGB; c) GND map of the same area. Higher density of dislocations is observed near the grain/laths boundaries while the density decreases in the inner part of the grains/laths; d) IQ map of the microstructure of the CHS. It can be observed that the average lath size is larger than that of the UFHS; e) The misorientation map of the CHS shows smaller amount of red grain boundaries while the; f) GND map of the CHS indicates lower dislocation density inside the martensite laths. 

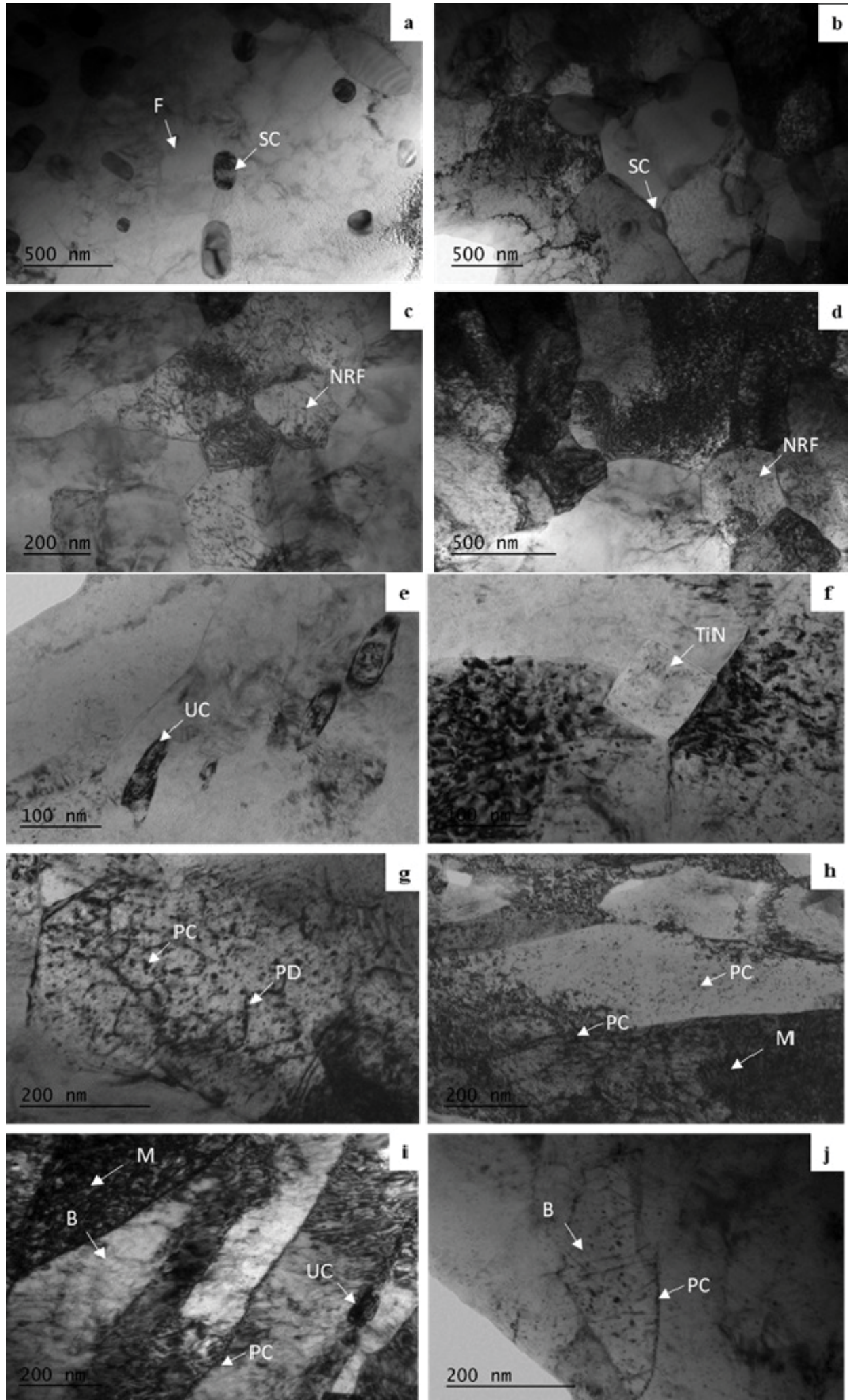

Figure 7: Bright field TEM images of the UFHS. a, b) The microstructure of the initial steel consisted of ferrite (F) and spheroidized cementite (SC); c, d) The microstructure consists of very fine non-recrystallized ferrite (NRF) grains as a result of the short heating time; e, f) Undissolved carbides (UC) and TiN are present in the microstructure as well due to lack of time for diffusion; $g$, h) Nano-carbides have been precipitated (PC) during the treatment. These carbides contribute on the pinning effect of the dislocations (PD); $i, j)$ The precipitation of oriented nano-carbides inside the laths and on the lath boundaries is an indication for the existence of bainitic ferrite $(B)$ in the microstructure. 
and Figures 5f, the martensite laths are very diverse concerning the lath size. Most importantly, in the SEM images, some morphologies were observed that seem similar to the representations of bainite of Takahashi and Bhadeshia [28] with carbides parallel to each other intercepting the lath (Figure $5 d$ and Figure 5f). To confirm these morphologies, TEM analysis had to be conducted.

\section{EBSD results}

EBSD analysis was conducted on the ultra-fast heat-treated specimen. The Image Quality (IQ) map (Figure 6a) shows the microstructure of a specific area of the UFHS. In this area, there is a great variety in the martensite lath size. The misorientation map (Figure 6b) of the same area indicates that most of the coarser martensite laths have formed after the heat treatment as they are surrounded by High Angle Grain Boundaries (HAGB > $15^{\circ}$ ). Low Angle Grain Boundaries are also present in a low scale. Red color indicates misorientation angles lower than $2^{\circ}$. TEM analysis showed that nano-sized, non-recrystallized ferrite grains were not able to transform during the heating because of the local microstructural constituents (Figure 7c). The GND map (Figure 6c) shows the effect of the presence of dislocations. The green color indicates higher density of dislocations while the blue color indicates lower density. The dislocation density is higher near the grain and lath boundaries while inside the grains/laths the density is lower. The density of GNDs is very high for the ultra-fast heat-treated steel. This is the result of the shift of the onset of recrystallization to higher temperatures [25] with ultra-fast heating. A shorter time at elevated temperatures during the ultra-fast heat treatment results in a less pronounced recovery of non-recrystallized ferritic grains and, thus, in a higher GND density compared to the conventional heat-treated condition [27]. Also, the martensite lath size contributes to higher concentration of dislocation. Finer laths translate to higher volume fraction of grain boundaries and therefore higher concentration sites with increased GND density. Comparing the EBSD results of the CHS sample, the average lath size is larger than that of the UFHS, as seen in Figure $6 \mathrm{~d}$. The misorientation map (Figure $6 e)$ of the same area shows fewer grain boundaries with orientation angle lower than $2^{\circ}$. Finally, the GND map of Figure $6 f$ indicates that the dislocation density is higher around the lath boundaries and much lower inside the laths.

EBSD results also confirm the diversity in lath size, mentioned in the SEM analysis. Figure 8 shows that almost the $50 \%$ of martensite laths have an av-

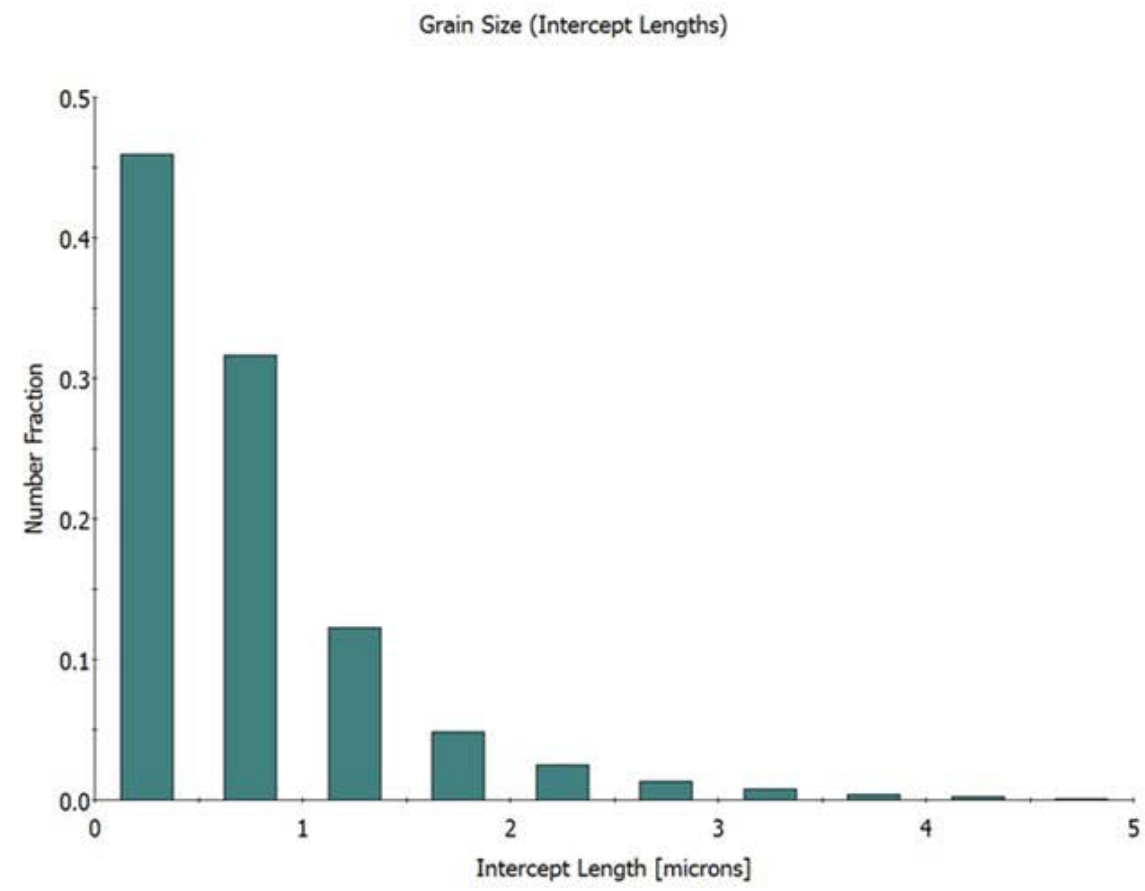

Chart Grain Size (Intercept Lengths)

Edge grains included in analysis as half grains 3095 grain intercepts sampled 104 Lines

Average Intercept length $=0.693$ microns

\begin{tabular}{ll}
\hline Intercept Length [microns] & Number Fraction \\
0.25 & 0.459451 \\
0.75 & 0.31664 \\
1.25 & 0.122456 \\
1.75 & 0.0481422 \\
2.25 & 0.0252019 \\
2.75 & 0.0132472 \\
3.25 & 0.00743134 \\
3.75 & 0.00420032 \\
4.25 & 0.00226171 \\
4.75 & 0.000969305 \\
& \\
Average & \\
Number & 0.693473 \\
Standard Deviation & \\
Number & 0.641331
\end{tabular}

Figure 8: The chart shows the intercept length of the martensite laths as a function of the number fraction \%. The majority of the laths has a size between 0.25 and $0.75 \mu \mathrm{m}$ indicating a very fine microstructure. 
erage size of $0.25 \mu \mathrm{m}, 30 \%$ have a size of $0.75 \mu \mathrm{m}$ and the rest $20 \%$ of the laths are larger than $1 \mu \mathrm{m}$. The average lath size was calculated from the EBSD analysis equal to $0.69 \mu \mathrm{m}$.

\section{TEM results}

The TEM analysis helps to better understand the microstructure evolution and the transformation phenomena that take place during ultra-fast heating. Figure $7 \mathrm{a}$ and Figure $7 \mathrm{~b}$ show the initial microstructure of the steel before the ultra-fast heat treatment. A ferritic matrix is clearly visible and there is a great number of spheroidized cementite. Concerning the UFHS, in Figure $7 c$ and Figure $7 d$, the varying grain and lath size can be seen. As reported earlier, this is the result of the lack of annealing time as some austenite grains cannot grow as much as others. This depends on the topical composition and the existence of undissolved carbides which do not provide enough space for austenite grains to grow [18] according to the massive transformation that takes place. The very fine grains are a result of the lack of heating time as there is no time for austenite to grow. The microstructure seen in these two figures consists of non-recrystallized ferrite grains that could not transform into austenite and martensite laths. Figure $7 \mathrm{e}$ and Figure $7 \mathrm{f}$ indicate the existence of the aforementioned undissolved $\mathrm{M}_{7} \mathrm{C}_{3}$ carbides [20,29] (Figure 7e) and Titanium nitrides (TiN) (Figure 7f). TEM analysis also showed increased density of dislocations. As seen in Figure $7 \mathrm{~g}$, most of these dislocations have been pinned (cannot move inside the material) because of the presence of nano-carbides. This phenomenon is called the pinning effect and has a positive impact on the tensile strength of the material [30]. The dislocation density is also reported from EBSD analysis that was performed. As was observed in the SEM images, the undissolved spheroidized cementite from the initial microstructure was observed as well during TEM analysis. In addition, much finer carbides were observed that have precipitated on the lath boundaries (Figure 7h, Figure $7 \mathrm{i}$ and Figure $7 \mathrm{j}$ ). These carbides do not originate from the initial microstructure and were precipitated during the ultra-fast thermal cycle. Also, some carbide-like morphologies were observed, parallel to each other intercepting the laths (Figure $7 \mathrm{j}$ ). As mentioned earlier, there are indications for the existence of bainitic ferrite in the microstructure. These TEM observations offer even more indications for the existence of bainitic ferrite. These bainitic morphologies can be seen in Figure $7 i$ and Figure $7 j$, where some laths are observed with small aligned precipitations intercepting them and even more nano-precipitates stacked on the laths boundaries. Laths with increased dislocation density that appear darker in the micrographs are characterized as martensitic laths whereas laths that appear brighter are characterized as bainitic [27].

\section{EDS results}

Composition analysis was conducted using STEM and EDS detectors mounted on the TEM microscope. Three points were chosen for the analysis (Figure 9). These points represent carbides that have not dissolved during the thermal treatment. The undissolved carbides act as nucleation sites, thus, leading to an exceptionally refined microstructure. Bouzouni and Papaefthymiou have studied thoroughly the kinetics of carbides dissolution during ultra-fast heating using Dictra and Thermocalc calculations [20]. Their simulation results indicate that the limited heating time prevents diffusion and the alloying elements segregate at carbide interfaces impeding their dissolution and leading to the formation of austenite with varying sizes and different carbon contents, which in some cases reach $1 \% \mathrm{wt}$. This means that it is possible to retain austenite at room temperature during quenching which has not been observed yet. This high carbon content in the carbides can also be seen in Spectrum 25 in which it reaches $0.86 \%$ wt. Moreover, the undissolved carbides as well as the segregation of alloying elements at grain boundaries affects phase transformations such as bainitic ferrite and martensite. The concentration of $\mathrm{Cr}$ and $\mathrm{Mn}$ is very high as expected from the steel's chemical composition. The low amount of Silicon (Si) content is responsible for carbide precipitation according to Li, et al. [31].

\section{Micro-hardness results}

Micro-hardness tests were also conducted on both samples. In order to obtain better results, the measurements with very high deviations were excluded. As seen in Figure 10, both steels have very high hardness values. Specifically, the ultra-fast heat-treated sample has an even higher average hardness value than the conventional heat-treated sample. The very high hardness of the CHS is expected because of the fully martensitic microstructure. 


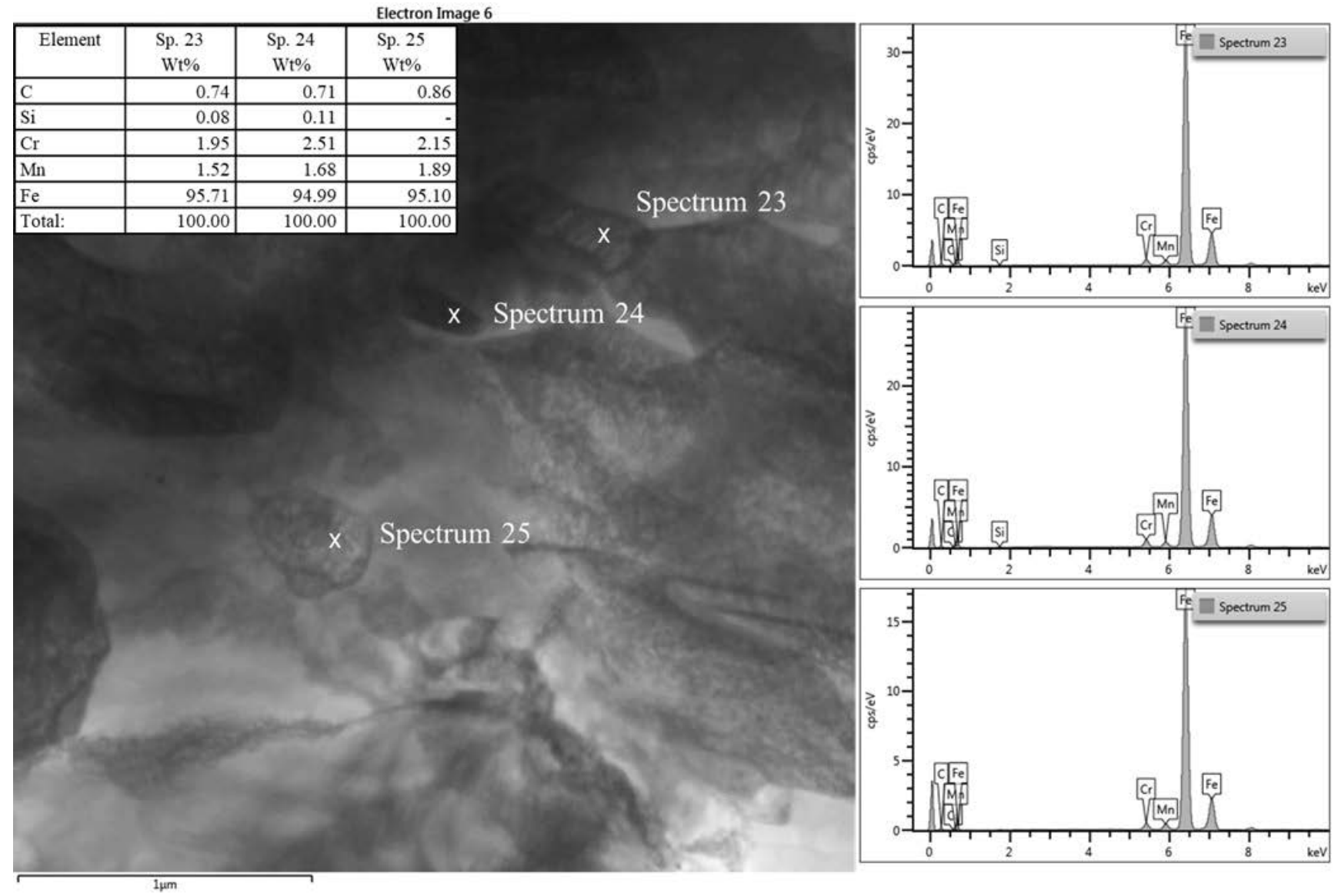

Figure 9: STEM image of the microstructure of the UFHS. The chemical analysis and spectrums of three different carbides are shown.

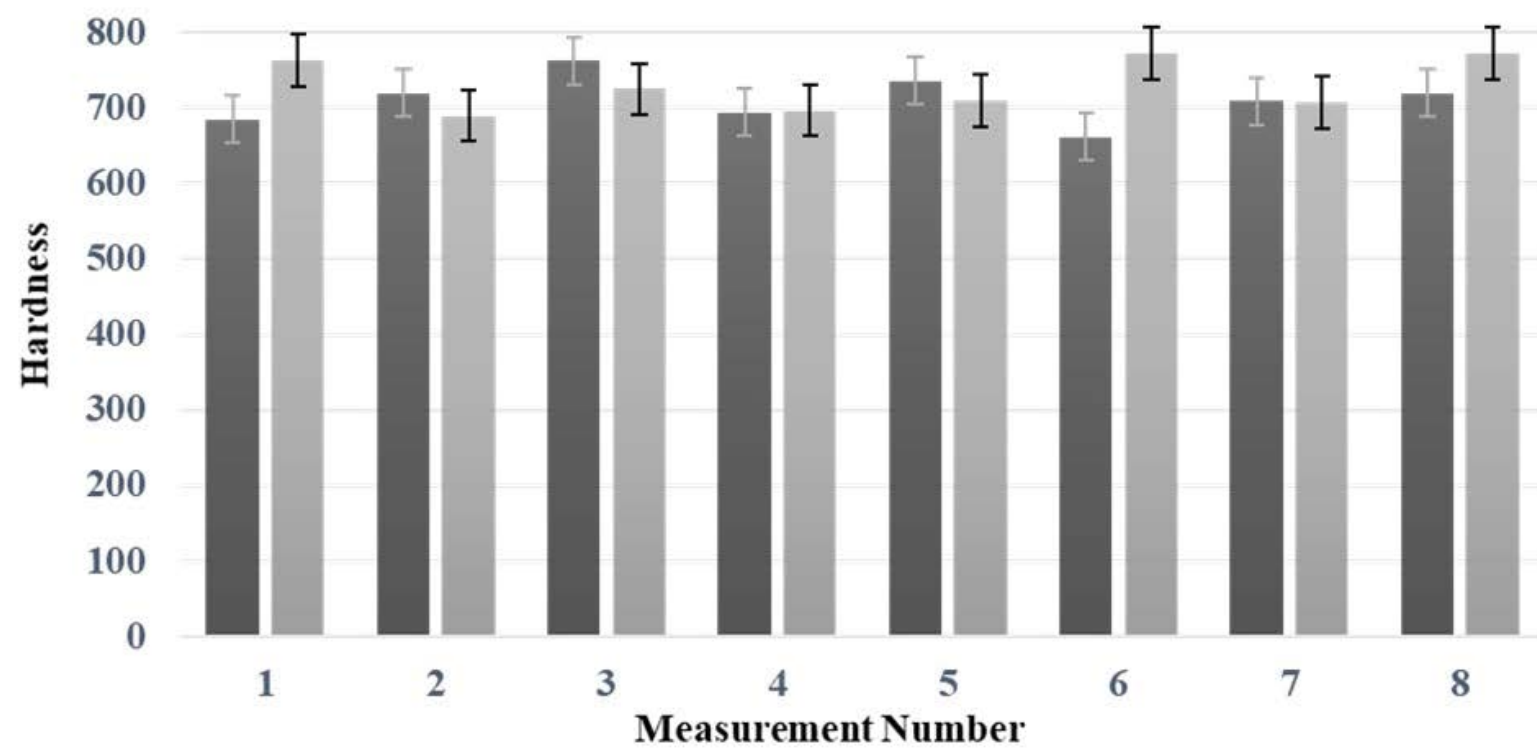

$$
\begin{array}{ll}
\text { ¿HS } & \text { Average: } 711 \\
=\text { UFHS } & \text { Average: } 730
\end{array}
$$

Standard Deviation: 31.4

Standard Deviation: $\mathbf{3 4 . 3}$

Figure 10: Micro-hardness results for the CHS and UFHS. Both steels have very high hardness values while the UFHS has a higher average hardness than the CHS. 
Concerning the UFHS, even though the microstructure has a lower volume fraction of martensite, the hardness is even higher. This can be the result of the undissolved carbides and pinned dislocations that affect positively the hardness of the sample, even though the microstructural constituents of this sample, such as non-recrystallized ferrite and bainite, have a lower hardness than martensite [32].

\section{Conclusion}

From the current research, it has been observed that the ultra-fast heating paves the way for a different microstructure evolution that results in a very refined mixed microstructure that improves strength and can also enhance ductility if austenite could be retained Dilatometry results have shown that there is an increase in the $A_{1}$ and $A_{3}$ transformation temperatures due to the increased heating rates. From the EBSD results, it can be statistically concluded that the ultra-fast heat-treated microstructure contains finer martensite and bainitic ferrite laths in comparison to the martensite laths present in the conventional heat-treated sample. The refinement of the microstructural components leads to an increase in the hardness of the UFH-treated material which is at the similar level to the $\mathrm{CH}$-treated material. Furthermore, the presence of undissolved carbides proves that there is no time for diffusion during the treatment and therefore the diffusion-controlled austenite formation and growth mechanism has been replaced by a massive transformation mechanism. EBSD and TEM results indicate the existence of dislocations in a large scale, which have a positive effect on the hardness of the steel via the pinning effect due to the undissolved carbides. Last but not least, TEM results provide strong evidence for the existence of bainitic ferrite as some bainitic morphologies have been observed. Bainitic ferrite occurs in the microstructure as a result of para-equilibrium transformations that take place in different areas of the specimen. It is important to understand that ultra-fast heat treatment results in a great heterogeneity within the microstructure and in variations of the local chemical composition.

\section{Acknowledgments}

For the provision of dilatometry samples, we thank Dr. ir. C. Goulas. Mrs. M. Bouzouni, cand. $\mathrm{PhD}$, is acknowledged for the thermodynamic calculations and the overall discussions on the subject.

\section{References}

1. T Lolla, GM Colla, B Narayanan, B Alexandrov, SS Babu (2011) Development of rapid heating and cooling (flash processing) process to produce high strength steel microstructures. Material Science and Technology 27: 863-875.

2. GM Cola (2015) Flash processing: Room temp stamping 1500-1600 MPa automotive structural components to $0 \mathrm{~T} / 1 \mathrm{~T}$ bend radii. Greater Columbus Convention Center, USA.

3. R Petrov, L Kestems (2016) Ultrafast heating of advanced high strength steels. Key note lecture at Thermec, Graz, Austria.

4. FM Castro Cerda (2017) Third generation advanced high strength steels via ultrafast heating. Doctoral Thesis, Ghent University.

5. R Petrov, F Hajyakbary, J Sidor, MJ Santofimia, J Sietsma, et al. (2012) Ultra-fast annealing of high strength steel. MTM International Virtual Journal 3: 5-8.

6. D De Knijf, A Puype, C Föjer, R Petrov (2015) The influence of ultra-fast annealing prior to quenching and partitioning on the microstructure and mechanical properties. Materials Science \& Engineering A 627: 182-190.

7. FM Castro Cerda, C Goulas, I Sabirov, S Papaefthymiou, A Monsalve, et al. (2016) Microstructure, texture and mechanical properties in a low carbon steel after ultrafast heating. Materials Science \& Engineering $A$ 672: 108-120.

8. C Wenquan, W Chang, W Cunyu, S Jie, W Maoqiu, et al. (2012) Microstructures and mechanical properties of the third-generation automobile steels fabricated by ARTannealing. Science China Technological Sciences 55: 1814-1822.

9. M Holzweissig, J Lackmann, S Konrad, S Mirko, N Thomas (2015) Influence of short austenitization treatments on the mechanical properties of low-alloy steels for hot forming applications. Metallurgical and Materials Transactions A 46: 3199-3207.

10. Lucas A, A De Paepe, H Petitgand, C Colin, L Chapuis, et al. (2007) Production of high quality formable grades in an ultra-short annealing processing line. Luxemburg.

11.R Petrov, J Sidor, L Kestens (2012) Texture formation in high strength low alloy steel reheated with ultrafast heating rates. Materials Science Forum 702-703: 798-801.

12. Y Meshkov, E Pereloma (2012) Phase transformations in steel: The effect of heating rate on reverse transformations in steels and Fe-Ni-based alloys. Phase Transformations in Steels 1: 581-618. 
13.FM Castro Cerda, B Schulz, S Papaefthymiou, A Artigas, A Monsalve, et al. (2016) The effect of ultrafast heating on cold-rolled low carbon steel: Formation and decomposition of Austenite. Metals 6: 321.

14.W Kaluba, R Taillard, J Foct (1998) The bainitic mechanism of austenite formation during rapid heating. Acta Materialia 46: 5917-5927.

15.H Aaronson, J Nie (2000) Discussion to 'The bainitic mechanism of austenite formation during rapid heating'. Scripta Materialia 42: 505-509.

16.W Kaluba, R Taillard, J Foct (2000) A reply to discussion to 'The bainitic mechanism of austenite formation during rapid heating'. Scripta Materialia 42: 511516.

17. Reis, L Bracke, R Petrov, WJ Kaluba, L Kestens (2003) Grain Refinement and texture change in interstitial free steels after severe rolling and ultra-short annealing. ISIJ International 43: 1260-1267.

18.A Puype (2014) Development of advanced high strength steel via ultrafast annealing. Master Thesis, Ghent University.

19.S Papaefthymiou, C Goulas, FM Castro Cerda, N Geerlofs, R Petrov (2017) The effect of heating rate on the microstructure of a soft-annealed medium carbon steel. Steel Research International 87.

20.M Bouzouni, S Papaefthymiou (2017) Preliminary study of carbide dissolution during an ultra-fast heat treatment in chromium molybdenum steel. International Journal of Metallurgy and Metal Physics 2.

21.V Savran (2004) Ferrite to austenite heating transformation in steels. NIMR Publication, Delft, Netherlands.

22.Goulas (2018) Developing novel heat treatments for automotive spring steels phase transformations, microstructure and performance. Doctoral Thesis, TU Delft.

23.FM Castro Cerda, F Vercruysse, C Goulas, B Schulz, RH Petrov (2018) 'Flash' annealing in a cold-rolled low carbon steel alloyed with $\mathrm{Cr}, \mathrm{Mn}, \mathrm{Mo}$, and $\mathrm{Nb}$ : Part I - continuous phase transformations. Steel Research International.
24.Kokosza, J Pacyna, P Bala, R Dziurka (2013) The influence of continuous heating rate on the austenite formation in the medium carbon trip steel. Archives of Materials Science and Engineering 62: 22-27.

25.M Valdes-Tabernero, F Vercruysse, I Sabirov, RH Petrov, MA Monclus, et al. (2018) Effect of ultrafast heating on the properties of the microconstituents in a low-carbon steel. Metallurgical and Materials Transactions A 49: 3145-3150.

26.R Dunlap (2012) The symmetry and packing fraction of the body centered tetragonal structure. European $\mathrm{J}$ of Physics Education 3.

27.S Papaefthymiou, M Bouzouni, R Petrov (2018) Study of carbide dissolution and austenite formation during ultra-fast heating in medium carbon chromium molybdenum steel. Metals 8: 646.

28. M Takahashi, H Bhadeshia (1990) Model for the transition from upper to lower bainite. Materials Science and Technology 6: 592-603.

29.M Bouzouni, S Papaefthymiou (2017) Modeling of the steel microstructure gained after the application of an ultra-fast heat treatment. Journal of Nanoscience with Advanced Technology 2: 15-19.

30.A Kamp, S Celotto, DN Hanlon (2012) Effect of tempering on the mechanical properties of high strength dual-phase steels. Materials Science and Engineering A 538: 35-41.

31.X Li, A Ramazani, U Prahl, W Bleck (2018) Quantification of complex-phase steel microstructure by using combined EBSD and EPMA measurements. Materials Characterization 142: 179-186.

32.L Ramos, D Matlock, G Krauss (1979) On the deformation behavior of dual-phase steels. Metallurgical Transactions A 10: 259-261. 